\title{
Borderline Ovarian Tumor and Pregnancy
}

\author{
Astrit M. GASHI ${ }^{1}$, Jakup ISMAJLI ${ }^{1}$, Xhevdet GOJNOVCI ${ }^{2}$ \\ ${ }^{1}$ Department of Obstetrics and Gynecology, University Clinical Centre of Kosovo, Pristine \\ ${ }^{2}$ Department of Neonatology Clinic, University Clinical Centre of Kosovo, Pristine
}

\begin{abstract}
We present a case of a 26 year-old pregnant woman, who was diagnosed a serous borderline tumor of the ovary, two years ago. CA 125, CA 19-9 and HE4 was within the normal range values. A Cystectomy was performed, where too histopathological diagnosis was decided. The patient was informed in detail about the nature of the disease and the treatment methods, and was assigned the time of surgical intervention (unilateral salpingo-oophorectomy with staging has been recommended), she does not accept any treatment method. After a year of follow-up with tumor markers, an exploratory laparoscopy with ovarian biopsy was performed. Left ovary was without histopathological changes, while the right ovary had histopathological changes for serous borderline tumor of the ovary, but without storm invasion. She decided for surgery. Fifteen days before surgery, she is presented for a routine visit, where pregnancy was confirmed. She was informed about potential risk, but categorically decided to continue the pregnancy. During pregnancy, there was no complication. She is presented for birth at 39+5 weeks of gestation. Due to obstetric status, which is not conducive to vaginal delivery and previous diagnoses, in co-think with the patient, it is decided that birth should be performed with Caesarean section. During a $C$-section, both ovaries are inspected, there are no macroscopic changes. Biopsy was obtained in both ovaries. The result was the same as two years ago. Because of the scarce studies, it is difficult to know which are the best management practices and especially to women who desire childbearing.
\end{abstract}

Keywords: borderline ovarian tumor, pregnancy, diagnosis, treatment

\section{INTRODUCTION}

Approximately $15 \%-20 \%$ of ovarian epithelial cancers are tumors of low malignant potential and usually have an excellent prognosis (1), with $95-99 \%$ long-term survival (2). The incidence of adnexal masses in pregnancy may be approximately $2 \%$ to $10 \%(3,5,6,7,8)$. Borderline ovarian tumors appear at any age, on average women affected are about the age of 40 . According to a study in Sweden, the incidence of borderline ovarian tumors has increased. This change may have been due to a rise in diagnostic activity, as well as by "a lack of protective effect of oral contraception use" (4).

In the histological aspect, borderline ovarian tumors differ from benign cysts by these charac- teristics; nuclear atypia, stratified epithelium, formation of microscopic papillary projections, cellular pleomorphism, mitotic activity. Are the 2 major histologic borderline ovarian tumor subtypes; serous and mucinous. Generally, borderline tumors have a slower growth rate than invasive ovarian carcinomas, and the cells of these epithelial tumors do not invade the stroma of the ovary. Borderline ovarian tumors, are difficult to detect clinically until they are advanced in size or stage. The most common symptoms are abdominal pain, abdominal distention, and abdominal mass, but they can also be altogether asymptomatic. The diagnosis of borderline ovarian cancer is based on transvaginal color Doppler ultrasonography (is useful in 
identifying the mass, detection of intratumoral blood flow and the resistance and pulsatility indexes), laboratory studies (Ca-125), computed tomography (CT) scans or MRI and on surgical staging.

Surgery is the primary treatment for women with borderline ovarian tumors. One of the initial considerations in planning surgery for borderline ovarian tumors is fertility-sparing surgery for patients who have not completed childbearing or who are young, her desire for future childbearing, and the degree of involvement of the ovaries (unilateral). Options for fertility-sparing surgery include ovarian cystectomy and unilateral anexectomy, with the surgical approach - minimally invasive or open technique.

\section{CASE PRESENTATION}

A 26-year-old woman, pregnant, presented for birth in the obstetric clinic at 39+5 weeks of gestation. Her obstetric and gynecologic history included; 4 years of infertility, a cystectomy two years ago and an exploratory laparoscopy with ovarian biopsy 1 year ago.

After an anamnesis focused on previous operations, it was understood that the patient two years ago had had a surgical intervention, where is removed a cyst in the right ovary, with dimensions 9x6×2.5 cm (Fig. 1).

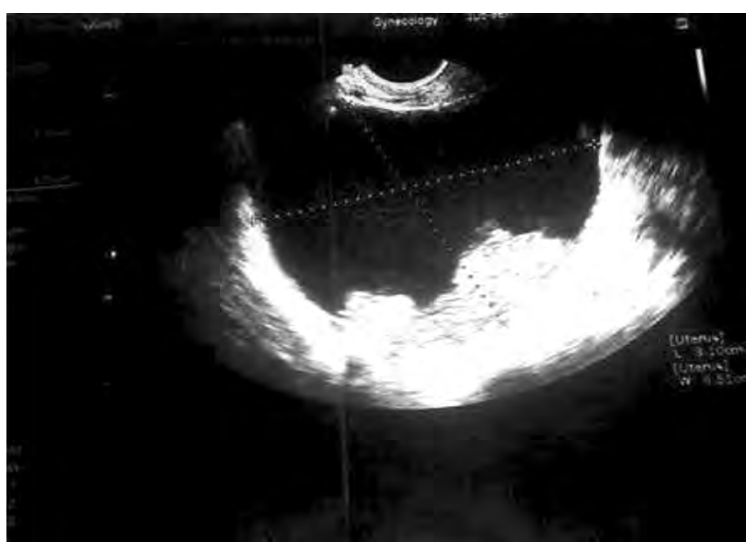

FIGURE 1. Ultrasound showing a complex mass, with a solid component and irregular.

In cutting, in the cyst lumen projected complex papillary structures covered with atypical epithelial cells. Histopathologic diagnosis was a serous borderline tumor of the ovary (ICD-O: 8462/1; C56.9)

The patient was followed with tumor markers as; Ca-125, Ca-19-9 and HE4, one year after the surgical treatment. Everything was normal.
After a year of follow-up with tumor markers, an exploratory laparoscopy with ovarian biopsy was performed. Left ovary was without histopathological changes, while the right ovary had histopathological changes for serous borderline tumor of the ovary, but without stromal invasion. The patient was informed in detail about the nature of the disease and the treatment methods, and was assigned the time of surgical intervention after two months.

Fifteen days before surgery, she is presented for a routine visit, where pregnancy was confirmed. She was informed about potential risk, but categorically decided to continue the pregnancy. During pregnancy, occasionally the size of the ovaries is ultrasound monitored. During pregnancy, there was no complication.

She is presented for birth at 39+5 weeks of gestation. Due to obstetric status, which is not conducive to vaginal delivery and previous diagnoses, in co-think with the patient, it is decided that birth should be performed with Caesarean section.

The patient gave birth to the infant of the female gender, with body weight 3600 grams and length $53 \mathrm{~cm}$, as well as APGAR score 8 .

Both ovaries are inspected, there are no macroscopic changes. Biopsy was obtained in both ovaries. The result was the same as two years ago. Left ovary was without histopathological changes, while the right ovary had histopathological changes for serous borderline tumor of the ovary, but without stromal invasion. Patient has been suggested to be removed also the right ovary along with the Caesarean section, but she does not accept for unfamiliar reasons.

\section{DISCUSSION}

Ovarian tumors of low malignant potential comprise $15-20 \%$ of all ovarian malignancies and usually have an excellent prognosis, with 95-99\% long-term survival $(1,2)$. Approximately one-third of borderline ovarian tumors are diagnosed in women at reproductive age (younger than 40 years old).

Borderline ovarian tumors, are difficult to detect clinically until they are advanced in size or stage. The most common symptoms are abdominal pain, abdominal distention, and abdominal mass, but they can also be altogether asymptomatic.

A general medical anamnesis, presenting symptoms, physical examination, imaging, labo- 
ratory studies, and family history can provide valuable insights in determining the appropriate treatment plan. Given this information, diagnosis can be established and establish an appropriate management plan.

Borderline ovarian tumors are frequently diagnosed during routine ultrasound exams. Through one pelvic ultrasound, we detect an adnexal mass and through one transvaginal color Doppler ultrasonography, identify features that have been associated with an increased risk of malignancy, such as size $>7 \mathrm{~cm}$, solid components, "complex" appearance, papillary structures, internal septations, bilaterality, irregular borders, ascites, increased vascularity, and low-resistance blood flow. Other help methods are computed tomography (CT) scans, MRI and surgical staging.

Also, serum tumor markers are useful in the evaluation of adnexal masses, including: CA125 , alpha-fetoprotein, lactate dehydrogenase, human chorionic gonadotropin, and inhibin $A$ and $B$, then also human epididymis 4 pro-tein (HE4), transthyretin, transferrin, $\beta$-microglobulin, and apolipoprotein $A 1$, which may improve preoperative assessment of the risk of malignancy in adnexal masses.

Earlier, radical surgery, respectively hysterectomy and bilateral salpingo-oophorectomy with peritoneal staging was standard regardless of the age of the patient, whereas today, unilateral salpingo-oophorectomy with or without staging has become the recommended management for women who desire childbearing.

\section{CONCLUSION}

We conclude that borderline ovarian tumors are lesions that have a slower growth rate and usually remain limited to ovaries for a long period of time. In our case even after two years, the stage remained the same, without storm invasion, without metastasis (clinical staging), and the woman achieved the goal of having a healthy child. Regardless that borderline ovarian tumors has an excellent prognosis, their diagnosis and management is often problematic, especially in women who desire childbearing.

\section{Ethic Committee approval}

This case report was approved by the Ethical Committee of the Faculty of Medicine, University of Prishtina, and the research was conducted in accordance with the Declaration of Helsinki guidelines. Written informed consent was obtained from the patient before inclusion in the study.

Conflict of interest: none declared

Financial support: none declared

\section{REFERENCES}

1. Seidman JD, Kurman RJ. Ovarian serous borderline tumors: a critical review of the literature with emphasis on prognostic indicators. Human pathology. 2000 May 1;31(5):539-57.

2. Fauvet $R$, Brzakowski M, Morice $P$, Resch B, Marret H, Graesslin O, Daraï E. Borderline ovarian tumors diagnosed during pregnancy exhibit a high incidence of aggressive features: results of a French multicenter study. Annals of oncology. 2011 Oct 14;23(6):1481-7. https://doi. org/10.1093/annonc/mdr452

3. N. Schwartz, I. E. Timor-Tritsch, and E. Wang, "Adnexal masses in pregnancy," Clinical Obstetrics and Gynecology, vol. 52, no. 4, pp. 570-585, 2009.
4. Skírnisdóttir I, Garmo H, Wilander E, Holmberg L. Borderline ovarian tumors in Sweden 1960-2005: trends in incidence and age at diagnosis compared to ovarian cancer. International journal of cancer. 2008 Oct 15;123(8):1897-901. https://doi. org/10.1002/ijc. 23724

5. Cavaco-Gomes J, Jorge Moreira C, Rocha A, Mota R, Paiva V, Costa A. Investigation and Management of Adnexal Masses in Pregnancy. Scientifica. 2016;2016. http:// dx.doi.org/10.1155/2016/3012802

6. Casanova J, Maciel R, Ferreira V, Fernandes E, Rodrigues RM. Borderline ovarian tumor during pregnancy: A case report. Case reports in obstetrics and gynecology. 2013;2013. http://dx.doi. org/10.1155/2013/160319

7. Alcazar JL, Olartecoechea B, Guerriero S, Jurado M. Expectant management of adnexal masses in selected premenopausal women: a prospective observational study. Ultrasound in Obstetrics \& Gynecology. 2013 May 1;41(5):582-8. https://doi. org/10.1002/uog.12369

8. Fruscio R, de Haan J, Van Calsteren K, Verheecke M, Mhallem M, Amant F. Ovarian cancer in pregnancy. Best Practice \& Research Clinical Obstetrics \& Gynaecology. 2017 May 1;41:108-17. https://doi.org/10.1016/j. bpobgyn.2016.09.013 